\title{
Temperature and Chemical Potential Dependence of the Parity Anomaly in Quantum Anomalous Hall Insulators
}

\author{
Christian Tutschku, ${ }^{1,4}$ Flavio S. Nogueira, ${ }^{2,4}$ Jeroen van den Brink, ${ }^{2,3,4}$ and E. M. Hankiewicz ${ }^{1,4}$ \\ ${ }^{1}$ Institute for Theoretical Physics, Julius-Maximilians-Universität Würzburg, 97074 Würzburg, Germany \\ ${ }^{2}$ Institute for Theoretical Solid State Physics, IFW Dresden, 01069 Dresden, Germany \\ ${ }^{3}$ Institute for Theoretical Physics, TU Dresden, 01069 Dresden, Germany \\ ${ }^{4}$ Würzburg-Dresden Cluster of Excellence ct.qmat
}

\begin{abstract}
The low-energy physics of two-dimensional Quantum Anomalous Hall insulators like ( $\mathrm{Hg}, \mathrm{Mn}) \mathrm{Te}$ quantum wells or magnetically doped $(\mathrm{Bi}, \mathrm{Sb})$ Te thin films can be effectively described by two Chern insulators, including a Dirac, as well as a momentum-dependent mass term. Each of those Chern insulators is directly related to the parity anomaly of planar quantum electrodynamics. In this work, we analyze the finite temperature Hall conductivity of a single Chern insulator in $2+1$ spacetime dimensions under the influence of a chemical potential and an out-of-plane magnetic field. At zero magnetic field, this non-dissipative transport coefficient originates from the parity anomaly of planar quantum electrodynamics. We show that the parity anomaly itself is not renormalized by finite temperature effects. However, it induces two terms of different physical origin in the effective action of a Chern insulator, which is proportional to the Hall conductivity. The first term is temperature and chemical potential independent, and solely encodes the intrinsic topological response. The second term specifies the non-topological thermal response of conduction and valence band states. In particular, we show that the relativistic mass of a Chern insulator counteracts finite temperature effects, whereas its non-relativistic mass enhances these corrections. Moreover, we extend our analysis to finite magnetic fields and relate the thermal response of a Chern insulator therein to the spectral asymmetry, which is a measure of the parity anomaly in orbital fields.
\end{abstract}

\section{INTRODUCTION}

Back in the 1980s, Haldane proposed the first solid state model of a Quantum Anomalous Hall (QAH) insulator by adding a parity-breaking [1] Dirac mass term to an otherwise gapless graphene structure [2]. Such a system features a non-zero Hall conductivity even in the absence of Landau levels (LLs). From a high-energy perspective, this model is directly related to the parity-anomaly of planar quantum electrodynamics, which implies that it is not possible to quantize a single Dirac fermion in a parity symmetric manner $[3,4]$. Strictly speaking, the Haldane model contains two Dirac fermions as it is based on the hexagonal lattice structure of graphene. However, by fine-tuning the Haldane mass, one of the Dirac fermion mass gaps can be closed, whereas the other one remains open. In this parameter limit, the band-structure contains a single gapless Dirac fermion with a non-zero Hall conductivity. This implies that in the Haldane model one of the Dirac fermions alone is suitable to realize the parity anomaly in $2+1$ dimensions. Hitherto, it was not possible to experimentally setup the Haldane model in a crystalline structure [5]. Instead, another type of QAH insulators was predicted in spin-polarized topological insulators (TIs) like $(\mathrm{Hg}, \mathrm{Mn}) \mathrm{Te}$ quantum wells $[6,7]$ or magnetically doped $(\mathrm{Bi}, \mathrm{Sb}) \mathrm{Te}$ thin films $[8,9]$. Their low-energy physics is captured by the superposition of two Chern insulators [10]. Similar to the Haldane model, the gap of one of these Chern insulators can be closed by magnetic doping of the system, whereas at the same time the second Chern insulator remains gapped. In this fine-tuned limit, the gapless Chern insulator realizes the parity anomaly as its contribution to the Hall conduc- tivity is in general non-zero. Hence, the analysis of single Chern insulators, which is the main purpose of the present work, allows to study measurable consequences of the parity anomaly in a solid state material. In contrast to the Dirac fermions in the Haldane model, each Chern insulator is characterized by two different paritybreaking mass terms: A conventional Dirac mass, as well as momentum-dependent Newtonian mass. As the Hall conductivity of a single Chern insulator is unaltered if one takes the parity-symmetric zero-mass limit, both, the Dirac as well as the momentum-dependent mass term, are directly related to the parity anomaly. It was recently shown that the momentum-dependent mass term acts similar to a Wilson fermion in a lattice regularization of a pure Dirac system [11]. As such, it ensures an integer- instead of a half-quantized Hall conductivity associated to a pure Dirac fermion $[12,13]$.

The parity anomaly is a zero magnetic field effect because an external magnetic field breaks the parity symmetry at the classical level. However, it can be shown that even in quantizing magnetic fields the signatures of the parity anomaly persist. They remain encoded in the spectral asymmetry [14]. Below a critical magnetic field, the parity anomaly effectively adds one LL to the entire Hall response of a Chern insulator. Above this field, the magnetic field closes the Dirac mass gap and the system exhibits a conventional QH response [15].

All these findings do not incorporate thermal effects. So far, finite temperature signatures in parity anomaly driven systems are restricted to pure Dirac models. Calculating the quantum effective action of these systems induces a temperature dependent and thus large gauge noninvariant Chern-Simons term originating from the par- 
ity anomaly [16-21]. While it was shown that this noninvariance is absorbed by higher order non-perturbative corrections to the effective action [22-29], this feature still gives rise to a fundamental question: Does the parity anomaly get renormalized by thermal effects? Answering this question is not only relevant for the QAH effect in the materials mentioned above. It is especially important in the case of interfaces between ferromagnetic insulators and three-dimensional topological insulators, where a proximity-induced interface magnetization has been experimentally observed at high temperatures [30, 31]. In this case the out-of-plane magnetization causes a gap opening in the interface Dirac spectrum, which induces a parity anomaly on the TI surface and a concomitant magnetoelectric torque in the Landau-Lifshitz equation [32-34]. A similar effect is expected to occur on the surface of the recently discovered antiferromagnetic TI $\mathrm{MnBi}_{2} \mathrm{Te}_{4}[35,36]$, where the gap in the surface Dirac spectrum is an intrinsic feature of the system.

By definition, the parity anomaly only implies the breakdown of parity symmetry at the quantum level. This dictates a certain form of the band-structure, which is temperature independent [37]. Hence, the parity anomaly cannot obtain any finite temperature correction. In contrast, the prefactor of the anomaly induced Chern-Simons term in the effective action corresponds to the finite temperature Hall conductivity. We calculate this non-dissipative transport coefficient for Chern insulators including both, a Dirac as well as a momentum-dependent mass. We studied these systems in the absence and presence of a magnetic field, as well as with and without particle-hole symmetry. This leads to the following results: (i) The parity anomaly induces a topological part in the Hall conductivity which is temperature as well as chemical potential independent and described by the Chern number. (ii) The non-quantized finite temperature and chemical potential corrections to the Hall conductivity also originate from the parity anomaly, since they also depend on the band-structure. However, they do not depend on its topology, being rather related to the temperature-dependent filling of the valence and conduction bands. As expected, an increasing Dirac mass counteracts finite temperature effects. On the other hand, we show that in the nontrivial phase an increasing Newtonian mass enhances the finite temperature corrections. (iii) In finite magnetic fields, the thermal LL response renormalizes the parity anomalous part of the Hall conductivity. In the Dirac mass gap it adds to the otherwise quantized parity anomaly related contribution.

This work is structured as follows: In Sec. II, we discuss the relation of magnetically doped two-dimensional TIs to the parity anomaly and compare these systems to the Haldane model. In this context, we analyze the bandstructure of Chern insulators in the absence and presence of an out-of-plane magnetic field and with, as well as without particle-hole symmetry. In Sec. III and Sec. IV, we analyze the parity-odd transport of a Chern insulator for a finite temperature and chemical potential, as well as for zero and finite magnetic fields, respectively. In Sec. V we summarize our results and give an outlook.

\section{PARITY ANOMALY IN A QAH SYSTEM BEYOND THE HALDANE MODEL}

In this work, we consider $2+1$ dimensional Chern insulators which are defined by two different mass terms: A momentum independent Dirac mass $m$, as well as a momentum dependent Newtonian mass term $B|\mathbf{k}|^{2}$. The Lagrangian of such an insulator is given by [38]

$$
\mathcal{L}=\bar{\psi}\left(A \gamma_{\mu} k^{\mu}-m+B k_{i} k^{i}\right) \psi,
$$

where $\psi$ and $\bar{\psi}=\psi^{\dagger} \gamma_{0}$ are the two-component Dirac spinor and its adjoint, $\gamma_{\mu}=\left(\sigma_{3}, \mathrm{i} \sigma_{2}, \mathrm{i} \sigma_{1}\right)$ are the $2+1 \mathrm{di}-$ mensional Dirac matrices, and we consider the metric $g^{\mu \nu}=\operatorname{diag}(+,-,-)$. Here and throughout the manuscript, Greek indices run over the space-time coordinates $\{0,1,2\}$, while roman indices run over the spatial components $\{1,2\}$ only. Moreover, the parameter $A$ is proportional to the Fermi velocity and $\sigma_{1,2,3}$ define the Pauli matrices. Notice, that in comparison to a pure Dirac Lagrangian, the additional Newtonian mass term in Eq. (1) breaks the Lorentz symmetry as it only involves spatial momenta.

The first-quantized Hamiltonian associated to Eq. (1) can be derived by a Legendre transformation:

$$
\mathcal{H}=A\left(k_{1} \sigma_{1}-k_{2} \sigma_{2}\right)+(m-B \alpha) \sigma_{3} .
$$

Here, we introduced the abbreviation $\alpha=k_{1}^{2}+k_{2}^{2}$. Both of the mass terms in Eq. (2), $m$ and $B \alpha$, break the parity symmetry of the Hamilton. In $2+1$ dimensions, parity symmetry is defined as invariance of the theory under $\mathcal{P}:\left(x_{0}, x_{1}, x_{2}\right) \rightarrow\left(x_{0},-x_{1}, x_{2}\right)$. Consequently, the Dirac as well as the Newtonian mass contribute to the integer Chern number [12, 13] [39]

$$
\mathcal{C}_{\mathrm{CI}}=(\operatorname{sgn}(m)+\operatorname{sgn}(B)) / 2 .
$$

Even in the parity symmetric limit $m, B \rightarrow 0^{ \pm}, \mathcal{C}_{\mathrm{CI}}$ does not vanish for $\operatorname{sgn}(m / B)>0$. This effect is known as the parity anomaly of Dirac-like systems in odd space-time dimensions. Initially, the parity anomaly has been predicted for a pure Dirac spectrum in Ref. [4]. Due to the absence of a Newtonian mass term, the Chern number of a pure Dirac system is given by $\mathcal{C}_{\mathrm{QED}}= \pm \operatorname{sgn}(m) / 2$ before regularization. Hence, it is half quantized and always non-zero. In contrast, the Chern number in Eq. (3) is integer quantized and defines two different phases: For $m / B>0$, the system is topologically nontrivial with $\mathcal{C}_{\mathrm{CI}}= \pm 1$, while for $m / B<0$, the system is topologically trivial with $\mathcal{C}_{\mathrm{CI}}=0$. In a solid state system with a crystal lattice, Dirac fermions in $2+1$ space-time dimensions always come in pairs [40]. The naive lattice discretization 


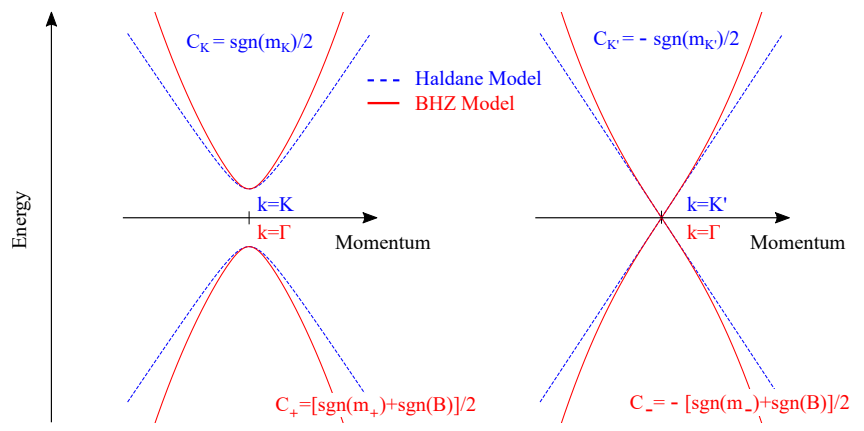

FIG. 1. Schematic illustration of how a single Dirac fermion or Chern insulator realizes the parity anomaly in the Haldane (blue) or BHZ (red) model. In the Haldane model, both Dirac fermions at the $K$ and $K^{\prime}$ points of the hexagonal lattice structure contribute $\pm 1 / 2$ to Chern number, whereas in the QAH phase of the BHZ model only one of the Chern insulators is topologically nontrivial and has a finite Chern number $C=$ \pm 1 . More explanations are given in the text.

of a pure Dirac fermion leads to a phenomenon called fermion-doubling, which predicts the existence of a second Dirac fermion of opposite Chern number at the edge of the lattice Brillouin zone. Thus, the entire system has Chern number zero and the parity anomaly of a single Dirac fermion cannot be measured. However, in his seminal work [2], Haldane found a way to circumvent this difficulty in a condensed matter system. He proposed a way of how to realize a single Dirac fermion in the bulk spectrum of graphene by separately manipulating the two Dirac gaps at the $K$ and $K^{\prime}$ points of the hexagonal lattice structure via a complex hopping parameter. In particular, this parameter allows to close only one of the Dirac gaps, whereas the other one remains open. Hence, his model suggests a way of how to realize a solid state system which has a single gapless Dirac fermion in $2+1$ dimensions but still a non-zero, integer Chern number $\mathcal{C}_{\mathrm{HM}}=\left(\operatorname{sgn}\left(\mathrm{m}_{\mathrm{K}}\right)-\operatorname{sgn}\left(\mathrm{m}_{\mathrm{K}^{\prime}}\right)\right) / 2$. Here, $m_{\mathrm{K}}$ and $m_{\mathrm{K}^{\prime}}$ are the Dirac mass terms at the $\mathrm{K}$ and $\mathrm{K}^{\prime}$ points in graphene, respectively.

While so far the Haldane model has not yet been realized in a solid state material, a closely related QAH effect has been predicted in two-dimensional systems like $(\mathrm{Hg}, \mathrm{Mn}) \mathrm{Te}$ quantum wells or magnetically doped $(\mathrm{Bi}, \mathrm{Sb}) \mathrm{Te}$ thin films. In the vicinity of the bulk gap, these systems can be effectively described by the BernevigHughes-Zhang (BHZ) model

$$
\mathcal{H}_{\mathrm{BHZ}}=\left(\begin{array}{cc}
\mathcal{H}_{\mathrm{CI}}^{+}(\mathbf{k}) & 0 \\
0 & \mathcal{H}_{\mathrm{CI}}^{-\star}(-\mathbf{k})
\end{array}\right)
$$

which consists of two copies of the Chern insulators defined in Eq. (2). They are decoupled [41] and the index \pm defines their (pseudo-)spin polarization

$$
\mathcal{H}_{\mathrm{CI}}^{ \pm}=A\left(k_{1} \sigma_{1}-k_{2} \sigma_{2}\right) \pm\left(m_{ \pm}-B \alpha\right) \sigma_{3}-D \alpha \sigma_{0} .
$$

In comparison to Eq. (2), Eq. (5) also includes a particlehole asymmetry $D \alpha \sigma_{0}$. Since this term is parity-even, it neither contributes to the parity anomaly nor changes the Chern number in Eq. (3) [42]. Therefore, let us first consider particle-hole symmetric systems with $D=0$. The Dirac masses $m_{ \pm}$of each (pseudo-)spin block in Eq. (5) can be tuned by magnetic doping of the system. It is in particular possible to drive one of the Chern insulators in the topologically trivial regime and to close, at the same time, the gap of the second non-trivial Chern insulator. Analogously to the Haldane model, in this scenario the single gapless Chern insulator alone realizes the parity anomaly of a Dirac-like system in $2+1$ dimensions. Schematically, this limit is illustrated in Fig. 1.

However, while in the Haldane model both Dirac fermions contribute $\pm 1 / 2$ to the Chern number, in the QAH phase of the BHZ model only one of the Chern insulators has Chern number $\mathcal{C}_{\mathrm{CI}}= \pm 1$. The other one is topologically trivial with $C_{\mathrm{CI}}=0$. Hence, studying the single Chern insulator in Eq. (2) is sufficient to analyze the consequences of the parity anomaly in experimentally realizable systems like $(\mathrm{Hg}, \mathrm{Mn}) \mathrm{Te}$ quantum wells or magnetically doped $(\mathrm{Bi}, \mathrm{Sb}) \mathrm{Te}$ thin films.

The spectrum associated to Eq. (2) is given by

$$
\epsilon^{ \pm}(\alpha)= \pm \sqrt{A^{2} \alpha+(m-B \alpha)^{2}},
$$

where \pm encodes the conduction and the valence band, respectively. In Fig. 2, we show the influence of the mass parameters on the band-structure. Depending on the values for $m, B$, and $A$, the band-structure changes significantly. For $m / B>0$, the system is topologically nontrivial with $\mathcal{C}_{\mathrm{CI}}= \pm 1$. The minimal gap can be either located at the $\Gamma$-point or at $\alpha_{\min }=\left(2 m B-A^{2}\right) /\left(2 B^{2}\right)$, corresponding to a camel-back structure. Thus, it is defined by $2|m|$ or by the absolute value of

$$
\Delta=A \sqrt{4 m B-A^{2}} / B .
$$

Increasing $|m|$ or $|B|$ in the nontrivial phase leads to a camel-back structure if $2 m B>A^{2}$, associated to $\alpha_{\min }>0$. The camel-back gap $|\Delta|$ increases with $m$ but decreases with $B$. For $4 m B=A^{2}, \Delta$ vanishes and the spectrum simplifies to,

$$
\epsilon^{ \pm}(\alpha)= \pm|(m+B \alpha)|
$$

For $m / B<0$, the system is topologically trivial. In this case the minimal gap is always located at the $\Gamma$-point.

If we include an out-of-plane magnetic field $H$, a LL spectrum forms if the magnetic length $l_{H}=\sqrt{\hbar /|e H|}$ is smaller than the system size [43]. For $s=\operatorname{sgn}(e H)$ and a finite particle-hole asymmetry $D$, one obtains

$$
\begin{aligned}
\epsilon_{n \neq 0}^{ \pm} & =-s \beta / 2-n \delta \pm \lambda_{n}, \\
\epsilon_{0} & =s(m-\beta / 2)-\delta / 2 .
\end{aligned}
$$

Here, we defined $\alpha=\sqrt{2} A / l_{H}, \beta=2 B / l_{H}^{2}, \delta=2 D / l_{H}^{2}$, and

$$
\lambda_{n}=\sqrt{\alpha^{2} n+(m-n \beta-s \delta / 2)^{2}} \text { with } n \in \mathbb{N}^{+} .
$$




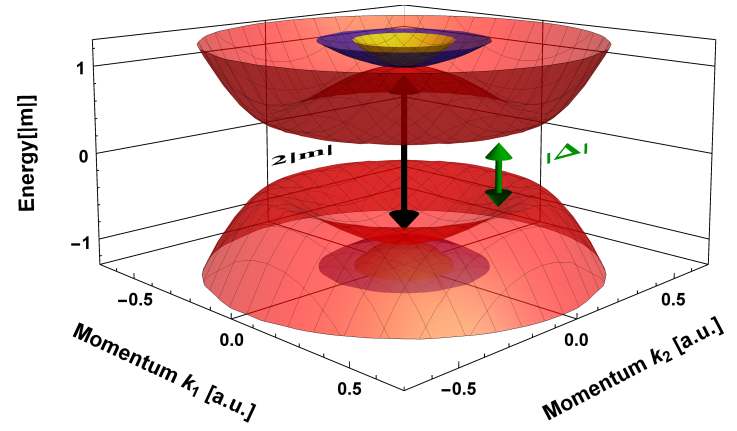

FIG. 2. Band-structure of a Chern insulator for zero magnetic field and $D=0$. Red and blue curves encode topologically non-trivial phases with $m=A=1$, and $B=5$ (red, camelback), or $m=1, A=3$, and $B=0.1$ (blue). The yellow curve corresponds to a topologically trivial phase with $m=1, A=5$, and $B=-0.1$. The minimal gap is either defined by $2|m|$ at the $\Gamma$-point or by $|\Delta|$ at $\alpha=\alpha_{\min }$, indicated by the black or green arrow, respectively.

As shown in Ref. [15], $H$ renormalizes the zero-field Chern number $\mathcal{C}_{\mathrm{CI}}$ in Eq. (3) to

$$
\mathcal{C}_{\mathrm{CI}}(H)=\left[\operatorname{sgn}\left(m-B / l_{H}^{2}\right)+\operatorname{sgn}(B)\right] / 2 .
$$

Hence, a magnetic field counteracts the parity anomaly related contribution to the Chern number, and closes the Dirac mass gap at $H_{\text {crit }}=\operatorname{sgn}(e H) m / B$. Beyond this critical magnetic field the parity anomaly vanishes.

This highly nontrivial statement deserves further clarification. The parity anomaly of a single Chern insulator is a zero magnetic field effect. It is directly related to the parity breaking elements of the zero-field Hamiltonian in Eq.(2) and its band-structure in Fig. 2. In quantizing magnetic fields, the Chern number of each LL only results from the magnetic field as it only depends on the magnetic length $l_{H}$. Nevertheless, the parity anomaly still has significant consequences in magnetic fields. Namely, it defines the Chern number in the Dirac mass gap, Eq. (12), resulting from the spectral asymmetry of the entire LL spectrum [15]. Since for $|H|>\left|H_{\text {crit }}\right|$, the spectral asymmetry vanishes, there are no measurable consequences of the parity anomaly beyond this critical value.

Above, we have introduced the concept of Chern insulators and have discussed their concrete relation to the parity anomaly in $2+1$ space-time dimensions. Next, we study finite temperature and density effects on the parity anomaly induced transport by calculating the Hall conductivity in zero, as well as in finite out-of-plane magnetic fields.

\section{ANOMALY INDUCED TRANSPORT IN ZERO MAGNETIC FIELD}

In what follows, we calculate the finite temperature Hall conductivity $\sigma_{\mathrm{xy}}$ corresponding to a particle-hole symmetric Chern insulator at zero magnetic field. This parity-odd and non-dissipative transport coefficient is directly related to the parity anomaly in $2+1$ space-time dimensions as it does not vanish in the parity symmetric limit $m, B \rightarrow 0^{ \pm}$. In our calculation, we disentangle topological from non-topological contributions to $\sigma_{\mathrm{xy}}$, the latter originating from thermal effects. For $D=0$, the finite temperature Hall conductivity of the Chern insulator in Eq. (2) is given by [44] [11]

$$
\sigma_{\mathrm{xy}}(T, \mu)=-\frac{e^{2}}{h} \int_{0}^{\infty} \mathrm{d} \alpha \frac{A^{2}(m+B \alpha)\left[f_{\mathrm{v}}(T, \mu)-f_{\mathrm{c}}(T, \mu)\right]}{4\left(A^{2} \alpha+(m-B \alpha)^{2}\right)^{3 / 2}},
$$

where $f_{\mathrm{c}, \mathrm{v}}(T, \mu)=\left[\mathrm{e}^{\left(\epsilon^{ \pm}(\alpha)-\mu\right) /\left(k_{\mathrm{B}} T\right)}+1\right]^{-1}$ are the conduction and valence band Fermi functions [20]. To disentangle topological from thermal contributions to $\sigma_{\mathrm{xy}}$, we use that

$$
f_{\mathrm{v}}(T, \mu)=1-\frac{\Theta(-\epsilon) \mathrm{e}^{(\epsilon-\mu) /\left(k_{\mathrm{B}} T\right)}}{\mathrm{e}^{(\epsilon-\mu) /\left(k_{\mathrm{B}} T\right)}+1},
$$

where $\epsilon(\alpha)$ encodes the entire spectrum and $\Theta$ is the Heaviside step function. With this identity, Eq. (13) decomposes into two building blocks,

$$
\sigma_{\mathrm{xy}}(T, \mu)=\sigma_{x y}^{0}+\sigma_{x y}^{1}(T, \mu)
$$

with

$$
\begin{gathered}
\sigma_{x y}^{0}=-\frac{e^{2}}{2 h}(\operatorname{sgn}(m)+\operatorname{sgn}(\mathrm{B})) \\
\sigma_{x y}^{1}(T, \mu)=\frac{e^{2}}{h} \int \mathrm{d} \alpha \frac{A^{2}(m+B \alpha) \operatorname{sgn}(\epsilon)}{4 \pi \epsilon^{3}} \\
\times\left(\frac{\Theta(\epsilon)}{\mathrm{e}^{(\epsilon-\mu) /\left(k_{\mathrm{B}} T\right)}+1}+\frac{\Theta(-\epsilon)}{\mathrm{e}^{-(\epsilon-\mu) /\left(k_{\mathrm{B}} T\right)}+1}\right) .
\end{gathered}
$$

Equation (16) encodes the topological part of the Hall conductivity. In contrast, Eq. (17) defines the corrections originating from a finite temperature and chemical potential. These non-topological and thus non-quantized corrections are based on particle-hole excitations of the conduction and valence band. To solve Eq. (17), we use the assumption of a particle-hole symmetric Chern insulator with $D=0$. In particular, this implies

$$
\sigma_{x y}^{1}(T, \mu)=\sigma_{x y}^{\text {corr }}(T, \mu)+\sigma_{x y}^{\text {corr }}(T,-\mu) .
$$

To determine $\sigma_{x y}^{\text {corr }}(T, \mu)$ in the energy space, we need to solve Eq. (6) for $\alpha$. Due to the possible camel-back structure, this leads to two solutions

$$
\alpha_{ \pm}=\alpha_{\min } \pm \frac{\sqrt{\epsilon^{2}-\Delta^{2}}}{|B|} \text { with } \frac{\mathrm{d} \alpha_{ \pm}}{\mathrm{d} \epsilon}= \pm \frac{\epsilon}{|B| \sqrt{\epsilon^{2}-\Delta^{2}}}
$$


With these identities, we find the correction

$$
\begin{aligned}
\sigma_{x y}^{\text {corr }}(T, \mu)= & \frac{e^{2}}{2 h} \Theta\left[\alpha_{\min }\right] \int_{|\Delta|}^{\sqrt{m^{2}}} \frac{A^{2}+\frac{2|B| \Delta^{2}}{\sqrt{\epsilon^{2}-\Delta^{2}}}}{B \epsilon^{2}\left(\mathrm{e}^{(\epsilon-\mu) / T}+1\right)} \mathrm{d} \epsilon \\
& +\frac{e^{2}}{4 h} \int_{\sqrt{m^{2}}}^{\infty} \frac{A^{2}+\frac{2|B| \Delta^{2}}{\sqrt{\epsilon^{2}-\Delta^{2}}}}{B \epsilon^{2}\left(\mathrm{e}^{(\epsilon-\mu) / T}+1\right)} \mathrm{d} \epsilon
\end{aligned}
$$

While in Eq. (19) the second term captures the correction from a monotonic band-structure, the first term encodes a possible camel-back correction. For $4 m B=A^{2}$ with $\Delta=0, \sigma_{x y}^{\text {corr }}(T, \mu)$ reduces to

$$
\sigma_{x y}^{\text {corr }}(T, \mu)=\frac{m e^{2}}{h} \int_{\sqrt{m^{2}}}^{\infty} \frac{A^{2}}{\epsilon^{2}\left(\mathrm{e}^{(\epsilon-\mu) /\left(k_{\mathrm{B}} T\right)}+1\right)} \mathrm{d} \epsilon,
$$

which is twice the $\mathrm{QED}_{2+1}$ result with $B=0$. Analogously to Eq. (16), the Newtonian mass provides a factor of two to the thermal corrections of the $\mathrm{QED}_{2+1}$ conductivity. For the solution in Eq. (20), we can define the corrections in terms of the Gamma function $\Gamma(x)$ and the reduced Fermi-Dirac integral $F_{j}(x, b)$ [App. A]. In total, this leads to

$$
\begin{gathered}
\sigma_{x y}^{1}(T, \mu)=\frac{e^{2}}{h} \sum_{s= \pm} \frac{A^{2} \Gamma(-1) F_{-2}\left(\frac{s \mu}{k_{\mathrm{B}} T}, \frac{|m|}{k_{\mathrm{B}} T}\right)}{k_{\mathrm{B}} T} \\
\stackrel{T \ll|m|}{=} \frac{e^{2}}{h} \frac{A^{2} \Gamma\left(-1, \frac{|m|}{k_{\mathrm{B}} T}\right)}{k_{\mathrm{B}} T} .
\end{gathered}
$$

In Eq. (21), we approximate the result for low temperatures in comparison to the gap and for zero chemical potential. $\Gamma(s, b)$ is the incomplete Gamma function [App. A].

The general correction in Eq. (19) cannot be expressed via the integral functions above since $\Delta \neq 0$. In Fig. 3, we plot the functional dependence of $\sigma_{\mathrm{xy}}(\mu, T)$ for different choices of $m$ and $B$. While increasing the Dirac mass always counteracts the temperature, increasing $B$ enhances temperature effects in the topologically nontrivial phase. As discussed below Eq. (7), this originates from the property that $B$ decreases the camel-back gap. Thus, both masses contribute equally to the topological part of the Hall conductivity in Eq. (16), while they counteract each other in the thermal corrections, Eq.(17), for $m / B>0$.

Notice, that even in the topologically trivial phase $m / B<0$ the system has a finite Hall conductivity in the Dirac mass gap [cf. Figs. 3(c) and (d)]. This is also directly related to the parity anomaly since it arises from the broken parity symmetry of the band-structure, which is independent of $\operatorname{sgn}(m / B)$. However, in the topologically trivial phase the Newtonian mass cannot generate a camel-back structure. In this case both, the Dirac and the Newtonian mass term counteract the finite temperature broadening of the Fermi-Dirac distribution.
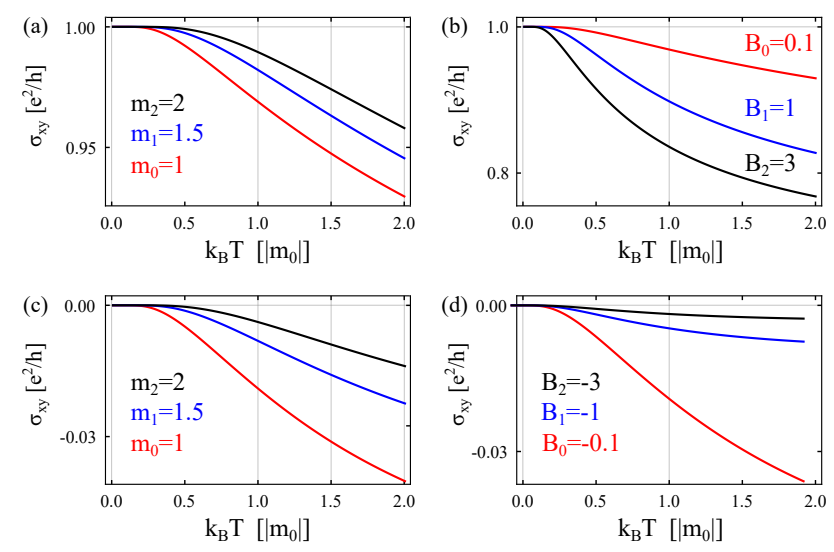

FIG. 3. Finite temperature Hall conductivity of a Chern insulator with $A=1$ and $D=0$. In (a) and (c), we vary the Dirac mass while $B= \pm 0.1$, respectively. In (b) and (d), we vary the Newtonian mass for $m=1$. In all sub-figures we consider zero chemical potential. Sub-figures (a) and (b) correspond to the the topologically non-trivial regime, while sub-figures (c) and (d) correspond to the topologically trivial regime.

\section{ANOMALY INDUCED TRANSPORT IN FINITE MAGNETIC FIELD}

Having analyzed a particle-hole symmetric Chern insulator at zero magnetic field, we now include a particlehole asymmetry and an out-of-plane magnetic field $H$, where the latter gives rise to the LL spectrum in Eq. (9). The Hall conductivity can be calculated by means of Streda's formula via the expectation value of the charge operator $\langle N\rangle_{T, \mu}[16,45]$, yielding

$$
\sigma_{\mathrm{xy}}(T, \mu)=-\frac{\partial\langle e N\rangle_{T, \mu}}{\partial H}=\frac{e}{2} \frac{\partial \eta_{H}}{\partial H}-\frac{\partial\left\langle e N_{0}\right\rangle}{\partial H} .
$$

Here, $\epsilon_{\mathrm{z}}=-m D / B$ is the charge neutrality point, and

$$
\begin{aligned}
& \eta_{\mathrm{H}}=\sum_{n} \operatorname{sgn}\left(\epsilon_{n}-\epsilon_{\mathrm{z}}\right)=-\frac{e H}{h}[\operatorname{sgn}(m-\beta / 2)+\operatorname{sgn}(B)], \\
& \left\langle N_{0}\right\rangle=\sum_{n} \operatorname{sgn}\left(\epsilon_{n}-\epsilon_{\mathrm{z}}\right)\left[\frac{\Theta\left(\epsilon_{n}-\epsilon_{\mathrm{z}}\right)}{\mathrm{e}^{\frac{\epsilon_{n}-\mu}{k_{\mathrm{B}} T}}+1}+\frac{\Theta\left(\epsilon_{\mathrm{z}}-\epsilon_{n}\right)}{\mathrm{e}^{-\frac{\epsilon_{n}-\mu}{k_{\mathrm{B}} T}}+1}\right] .
\end{aligned}
$$

The spectral asymmetry $\eta_{\mathrm{H}}$ counts the difference in the number of conduction and valence band states. Therefore, as long as the band structure is not changed, it is temperature and chemical potential independent and solely carries the information of the topological contribution of the parity anomaly to $\sigma_{\mathrm{xy}}$ in magnetic fields. This enables the connection between the Hall conductivity and the parity anomaly even at finite magnetic fields. In contrast, $\left\langle N_{0}\right\rangle$ encodes the thermal LL response, as it defines the thermal occupation of the valence and the conduction band. Due to the associated flat dispersion relation, this response entirely originates from the magnetic field topology and no more from the parity anomaly. All LLs with $n \in \mathbb{N}^{+}$come in pairs. With the degeneracy 
$|e H| / h$, their contribution to the charge operator is given by

$$
\left\langle N_{0}\right\rangle_{n \neq 0}=\frac{|e H|}{h} \sum_{n \neq 0, s= \pm} \frac{s}{\mathrm{e}^{\frac{s\left(e_{n}^{s}-\mu\right)}{k_{\mathrm{B}} T}}+1} .
$$

Additionally to this conventional LL response for finite $\mu$ and $T$, the zero LL also needs to compensate its contribution to $\eta_{\mathrm{H}}$ outside of the Dirac mass gap. In particular, it needs to cancel the term $\propto \operatorname{sgn}(m-\beta / 2)$ in Eq. (23) for $|\mu+\delta / 2|>|m-\beta / 2|[15]$. Since the zero LL can either be part of the conduction or of the valence band, we can simplify its contribution to $\left\langle N_{0}\right\rangle$. By using the properties of the hyperbolic tangent, we find for the zero LL with $n=0$

$$
\begin{gathered}
\left\langle N_{0}\right\rangle_{0}=-\frac{|e H| \operatorname{sgn}\left(\epsilon_{0}-\epsilon_{\mathrm{z}}\right)}{2 h}\left[\Theta\left(\epsilon_{0}-\epsilon_{\mathrm{z}}\right)\left[\tanh \left(\frac{\epsilon_{0}-\mu}{2 k_{\mathrm{B}} T}\right)-1\right]\right. \\
\left.-\Theta\left(\epsilon_{\mathrm{z}}-\epsilon_{0}\right)\left[\tanh \left(\frac{\epsilon_{0}-\mu}{2 k_{\mathrm{B}} T}\right)+1\right]\right] .
\end{gathered}
$$

This expression can be simplified further via the identities

$$
\begin{aligned}
\operatorname{sgn}\left(\epsilon_{0}-\epsilon_{\mathrm{z}}\right) & =\operatorname{sgn}\left(\epsilon_{0}+\delta / 2\right)=\operatorname{sgn}(e H) \operatorname{sgn}(m-\beta / 2) \\
\Theta\left(\epsilon_{0}-\epsilon_{\mathrm{z}}\right)-\Theta\left(-\epsilon_{0}+\epsilon_{\mathrm{z}}\right) & =\operatorname{sgn}\left(\epsilon_{0}-\epsilon_{\mathrm{z}}\right) \\
\Theta\left(\epsilon_{0}-\epsilon_{\mathrm{z}}\right)+\Theta\left(-\epsilon_{0}+\epsilon_{\mathrm{z}}\right) & =1 .
\end{aligned}
$$

Eventually, this implies the zero LL contribution

$$
\left\langle N_{0}\right\rangle_{0}=\frac{|e H|}{2 h}\left[\operatorname{sgn}(e H) \operatorname{sgn}(m-\beta / 2)-\tanh \left(\frac{\epsilon_{0}-\mu}{2 k_{\mathrm{B}} T}\right)\right],
$$

which reduces for $T \rightarrow 0$ and $\bar{\mu}=\mu+D / l_{H}^{2}$ to [App. A]

$$
\begin{aligned}
\left\langle N_{0}\right\rangle_{0} & =\frac{|e H|}{2 h} \Theta(|\bar{\mu}|-|m-\beta / 2|) \\
& \times[\operatorname{sgn}(e H) \operatorname{sgn}(\mathrm{m}-\beta / 2)+\operatorname{sgn}(\bar{\mu})] .
\end{aligned}
$$

For $T=0$, the zero LL contribution to $\eta_{\mathrm{H}}$ clearly gets compensated outside of the Dirac mass gap. As expected, finite temperature effects soften this property.

In Fig. 4, we used Eq. (22) to connect the charge operator to $\sigma_{\mathrm{xy}}$, and plotted Hall conductivity corresponding to the parity anomaly and to each LL, separately. Moreover, we show the entire signal as a function of the chemical potential. While the Hall conductivity contribution related to the parity anomaly is $T$ and $\mu$ independent, each LL comes along with an exponentially suppressed temperature broadening. Consequently, all LLs contribute to the Hall conductivity in the Dirac mass gap. This renormalizes the zero temperature violation of the Onsager relation [46] discussed recently in Ref. [15]. Last but not least, let us emphasize that the Hall plateau originating from the parity anomaly is much more robust than LL plateaus with respect to finite temperature effects. Due

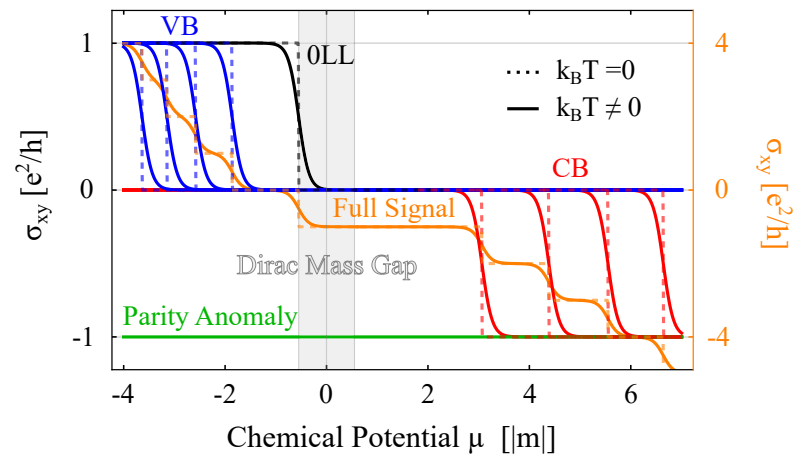

FIG. 4. Finite temperature Hall conductivity of a Chern insulator in a magnetic field $H=3 T$ with: $A=1, m=-1$, $B=-0.1$, and $D=-0.05$. The response of each valence (blue) and conduction band (red) LL is shown separately. The zero LL response is illustrated in black, the parity anomaly related contribution is depicted in green. The combined signal is shown in orange. Dashed lines correspond to $k_{\mathrm{B}} T=0$, solid lines are associated to $k_{\mathrm{B}} T=0.01$. The Dirac mass gap is shown in grey.

to the lack of a zero-LL partner, the parity anomaly response is approximately unaltered [47] until the chemical potential comes close to the $n=1$ conduction or valence band LL, depending on the sign of the magnetic field. Quantitatively, this means

$$
\left|\epsilon_{1}^{+}-\epsilon_{0}\right|>\left|\epsilon_{n+1}^{ \pm}-\epsilon_{n}^{ \pm}\right| \quad \forall n \in \mathbb{N}^{+},
$$

assuming that the zero-LL is part of the valence band [cf. Fig. 4]. Therefore, finite temperature effects firstly smear out the LL steps before they eventually prevent any quantization for the finite temperature Hall conductivity.

\section{SUMMARY AND OUTLOOK}

In this work we analyzed the finite temperature Hall conductivity of two-dimensional Chern insulators under the influence of a chemical potential and an out-of-plane magnetic field. At zero magnetic field, this quantity originates from the parity anomaly. As such, we were able to show that the parity anomaly is not renormalized by finite temperature effects. Instead, it induces two terms of different physical origin in the effective action of a Chern insulator, which are proportional to its Hall conductivity. The first term is temperature and chemical potential independent, solely encoding the intrinsic topological response. The second term specifies the non-topological thermal response of conduction and valence band states. We showed that in the topologically nontrivial phase, an increasing relativistic mass term of a Chern insulator counteracts finite temperature effects, whereas an increasing non-relativistic mass term enhances these corrections. In contrast, both mass terms counteract the finite temperature broadening 
of the Fermi-Dirac distribution in the topologically trivial phase, as the Newtonian mass cannot cause a camel-back gap in this case. In magnetized II-VI QAH insulators, like ( $\mathrm{Hg}, \mathrm{Mn}) \mathrm{Te}$ quantum wells, these parameters can be tuned by changing the quantum well width, or by changing the concentration of the magnetic dopants. Moreover, we derived the thermal response of a Chern insulator in a magnetic field and clarified its relation to the spectral asymmetry $\eta_{\mathrm{H}}$. This quantity is a measure of the parity anomaly in magnetic fields. In particular, we derived in which way the thermal LL response renormalizes the parity anomalous part of the Hall conductivity in magnetic fields. Especially in the Dirac mass gap, this response adds to the otherwise quantized and temperature independent part of the Hall conductivity arising from the parity anomaly. We showed that the anomalous part of the Hall response in the Dirac mass gap is much more robust than the common LL contributions with respect to finite temperature effects. Our findings should be experimentally verifiable in $\mathrm{QAH}$ insulators such as $(\mathrm{Hg}, \mathrm{Mn}) \mathrm{Te}$ quantum wells, magnetically doped $(\mathrm{Bi}, \mathrm{Sb}) \mathrm{Te}$ thin films, or bilayer structures of three-dimensional topological and ferromagnetic insulators.

In the future, it would be interesting to extend this analysis to different anomalies in various space-time dimensions. For instance, the chiral, gravitational, and conformal anomaly should not depend on thermal effects, whereas they necessarily induce a temperature dependence at the effective action level.

\section{ACKNOWLEDGMENTS}

We thank J. Boettcher and R. Meyer for useful discussions. We acknowledge financial support through the Deutsche Forschungsgemeinschaft (DFG, German Research Foundation), project-id 258499086 - SFB 1170 'ToCoTronics' and SFB 1143 project A5, the ENB Graduate School on Topological Insulators and through the Würzburg-Dresden Cluster of Excellence on Complexity and Topology in Quantum Matter - ct.qmat (EXC 2147, project-id 39085490).
[1] Instead of a parity-even Dirac mass term, initially studied by Semenoff in Ref. [48].

[2] F. D. M. Haldane, Phys. Rev. Lett. 61, 2015 (1988).

[3] A. J. Niemi and G. W. Semenoff, Phys. Rev. Lett. 51, 2077 (1983).

[4] A. N. Redlich, Phys. Rev. Lett. 52, 18 (1984).

[5] So far, this model has been only realized in optical lattices $[49,50]$.

[6] C.-X. Liu, X.-L. Qi, X. Dai, Z. Fang, and S.-C. Zhang, Phys. Rev. Lett. 101, 146802 (2008).

[7] X.-L. Qi, T. L. Hughes, and S.-C. Zhang, Phys. Rev. B 78, 195424 (2008).

[8] R. Yu, W. Zhang, H.-J. Zhang, S.-C. Zhang, X. Dai, and Z. Fang, Science 329, 61 (2010).

[9] C.-Z. Chang, J. Zhang, X. Feng, J. Shen, Z. Zhang, M. Guo, K. Li, Y. Ou, P. Wei, L.-L. Wang, Z.-Q. Ji, Y. Feng, S. Ji, X. Chen, J. Jia, X. Dai, Z. Fang, S.-C. Zhang, K. He, Y. Wang, L. Lu, X.-C. Ma, and Q.-K. Xue, Science 340, 167 (2013).

[10] B. A. Bernevig, T. L. Hughes, and S.-C. Zhang, Science 314, 1757 (2006).

[11] C. Tutschku, J. Böttcher, R. Meyer, and E. M. Hankiewicz, Phys. Rev. Research 2, 033193 (2020).

[12] H.-Z. Lu, W.-Y. Shan, W. Yao, Q. Niu, and S.-Q. Shen, Phys. Rev. B 81, 115407 (2010).

[13] X.-L. Qi and S.-C. Zhang, Rev. Mod. Phys. 83, 1057 (2011).

[14] A. J. Niemi and G. W. Semenoff, Phys. Rev. D 30, 809 (1984).

[15] J. Böttcher, C. Tutschku, L. W. Molenkamp, and E. M. Hankiewicz, Phys. Rev. Lett. 123, 226602 (2019).

[16] A. J. Niemi, Nucl. Phys. B 251, 155 (1985).

[17] G. Dunne, K. Lee, and C. Lu, Physical Review Letters 78, 3434âĂŞ3437 (1997).
[18] G. V. Dunne, in Topological Aspects of Low-dimensional Systems: Proceedings, Les Houches Summer School of Theoretical Physics, Session 69: Les Houches, France, July 7-31 1998 (1998) arXiv:hep-th/9902115.

[19] A. Sissakian, O. Shevchenko, and S. Solganik, Nuclear Physics B 518, 455âĂŞ472 (1998).

[20] F. S. Nogueira and I. Eremin, Phys. Rev. B 90, 014431 (2014)

[21] C.-T. Ma, Fortschritte der Physik 66, 1800045 (2018).

[22] S. Deser, L. Griguolo, and D. Seminara, Physical Review Letters 79, 1976âĂŞ1979 (1997).

[23] C. D. Fosco, G. L. Rossini, and F. A. Schaposnik, Physical Review Letters 79, 1980âĂŞ1983 (1997).

[24] C. D. Fosco, G. L. Rossini, and F. A. Schaposnik, Physical Review D 56, 6547âĂŞ6555 (1997).

[25] M. Hott and G. Metikas, Phys. Rev. D 60, 067703 (1999).

[26] L. Salcedo, Nuclear Physics B 549, 98âĂŞ144 (1999).

[27] C. D. Fosco, G. L. Rossini, and F. A. Schaposnik, Phys. Rev. D 59, 085012 (1999).

[28] C. G. Beneventano, P. Giacconi, E. M. Santangelo, and R. Soldati, Journal of Physics A: Mathematical and Theoretical 42, 275401 (2009).

[29] C. D. Fosco and F. A. Schaposnik, Phys. Rev. D 95, 105011 (2017).

[30] F. Katmis, V. Lauter, F. S. Nogueira, B. A. Assaf, M. E. Jamer, P. Wei, B. Satpati, J. W. Freeland, I. Eremin, D. Heiman, P. Jarillo-Herrero, and J. S. Moodera, Nature 533, 513 (2016).

[31] C. Tang, C.-Z. Chang, G. Zhao, Y. Liu, Z. Jiang, C.-X. Liu, M. R. McCartney, D. J. Smith, T. Chen, J. S. Moodera, and J. Shi, Science Advances 3, e1700307 (2017).

[32] T. Yokoyama, J. Zang, and N. Nagaosa, Phys. Rev. B 81, 241410 (2010).

[33] F. S. Nogueira and I. Eremin, Phys. Rev. Lett. 109, 
237203 (2012)

[34] Y. Tserkovnyak and D. Loss, Phys. Rev. Lett. 108, 187201 (2012).

[35] M. M. Otrokov, I. I. Klimovskikh, H. Bentmann, D. Estyunin, A. Zeugner, Z. S. Aliev, S. Gaß, A. U. B. Wolter, A. V. Koroleva, A. M. Shikin, M. Blanco-Rey, M. Hoffmann, I. P. Rusinov, A. Y. Vyazovskaya, S. V. Eremeev, Y. M. Koroteev, V. M. Kuznetsov, F. Freyse, J. Sánchez-Barriga, I. R. Amiraslanov, M. B. Babanly, N. T. Mamedov, N. A. Abdullayev, V. N. Zverev, A. Alfonsov, V. Kataev, B. Büchner, E. F. Schwier, S. Kumar, A. Kimura, L. Petaccia, G. Di Santo, R. C. Vidal, S. Schatz, K. Kißner, M. Ünzelmann, C. H. Min, S. Moser, T. R. F. Peixoto, F. Reinert, A. Ernst, P. M. Echenique, A. Isaeva, and E. V. Chulkov, Nature 576, 416 (2019).

[36] R. C. Vidal, A. Zeugner, J. I. Facio, R. Ray, M. H. Haghighi, A. U. B. Wolter, L. T. Corredor Bohorquez, F. Caglieris, S. Moser, T. Figgemeier, T. R. F. Peixoto, H. B. Vasili, M. Valvidares, S. Jung, C. Cacho, A. Alfonsov, K. Mehlawat, V. Kataev, C. Hess, M. Richter, B. Büchner, J. van den Brink, M. Ruck, F. Reinert, H. Bentmann, and A. Isaeva, Phys. Rev. X 9, 041065 (2019).

[37] Rigorously, this statement is only true for small temperatures. For very large temperatures the system can deform, which essentially changes the band-structure. However, this scenario is beyond the scope of our analysis.

[38] Notice, that a similar Lagrangian can be used for the description of $2+1$ dimensional superfluid Fermi liquids, as discussed by G. E. Volovik in Ref. [51].

[39] Adding higher-order momentum dependent mass corrections to the Hamiltonian of a Chern insulator changes its Chern number. To respect the Galilean invariance of the system, any additional mass correction needs to be of even order in momentum. Essentially, the prefactor of the highest order mass correction replaces the Newtonian mass parameter $B$ in the Chern number Eq. (3). Since higher order mass corrections do change the bandcurvature, they will also alter the nonquantized Hall response at finite temperatures and chemical potentials. However, they will only change this response quantitatively. In particular, they do not prevent the possible gap closing apart from the $\Gamma$-point which drives the lowenergy response studied in this manuscript [cf. Eq.(19)].

[40] If the $2+1$ dimensional manifold is the boundary theory of a $3+1$ dimensional bulk, there could also be an odd number of Dirac fermions living at the boundary. For instance, this is the case at the boundary of $3+1$ dimensional topological insulators. However, we are not considering such systems but focus our analysis on pure $2+1$ dimensional bulk materials.

[41] For the scope of this work, bulk inversion asymmetry terms are unimportant. Therefore, they are neglected throughout the manuscript.

[42] J. Böttcher, C. Tutschku, and E. M. Hankiewicz, Phys. Rev. B 101, 195433 (2020).

[43] M. König, H. Buhmann, L. W. Molenkamp, T. Hughes, C.-X. Liu, X.-L. Qi, and S.-C. Zhang, Journal of the Physical Society of Japan 77, 031007 (2008).

[44] This result is obtained by calculating the vacuum polarization operator or, analogously, the current-current correlation function. In the language of quantum field theory, this corresponds to the evaluation of a one-loop Feynman diagram, whereas in the solid-state community Eq.(13) results from the Kubo-formalism.

[45] P. Streda, Journal of Physics C: Solid State Physics 15, L717 (1982).

[46] At $T=0$, the only contribution to the Hall conductivity in the Dirac mass gap is given by the parity anomaly in terms of the spectral asymmetry $\eta_{H}$ [cf. Eq. (22)]. Due to Eq. (23) this contribution clearly violates the Onsager relation defined as $\sigma_{\mathrm{xy}}(-H)=-\sigma_{\mathrm{xy}}(H)$. In contrast, this identity is fulfilled by any (thermal) LL contribution.

[47] Due to the exponential suppression of all LL contributions in the Dirac mass gap.

[48] G. W. Semenoff, Phys. Rev. Lett. 53, 2449 (1984).

[49] J. Xu, Q. Gu, and E. J. Mueller, Phys. Rev. Lett. 120, 085301 (2018).

[50] W. Liu, Z. Lin, Z. D. Wang, and Y. Chen, Sci. Rep. 8, 12898 (2018).

[51] G. E. Volovik, Soviet Physics - JETP (English Translation) 67, 1804 (1988).

\section{Appendix A: Appendix}

The reduced Fermi-Dirac integral is defined by

$$
F_{j}(x, b)=\frac{1}{\Gamma(j+1)} \int_{b}^{\infty} \mathrm{d} t \frac{t^{j}}{\mathrm{e}^{t-x}+1} .
$$

The incomplete $\Gamma$-function is defined by

$$
\Gamma(s, b)=\int_{b}^{\infty} \mathrm{d} t t^{s-1} e^{-t} .
$$

Let us comment on how to derive Eq. (27) from Eq. (26). In the zero temperature limit, the hyperbolic tangent in Eq. (27) becomes a sign-function, $\lim _{T \rightarrow 0} \tanh (x / T)=$ $\operatorname{sgn}(x)$. Due to this property, we need to distinguish two cases. The chemical potential is either located inside $(i)$ or outside $(\mathrm{ii})$ of the Dirac mass gap:

$$
\begin{gathered}
\text { (i) } \quad|\mu+\delta / 2|<|m-\beta / 2| \\
\text { (ii) } \quad|\mu+\delta / 2|>|m-\beta / 2| .
\end{gathered}
$$

For case $(i)$, the hyperbolic tangent in Eq. (27) reduces to $\operatorname{sgn}(e H) \operatorname{sgn}(m-\beta / 2)$ and consequently leads to $\left\langle N_{0}\right\rangle_{0}=$ 0 . Instead, for case $(i i)$, it reduces to $\operatorname{sgn}(\mu+\delta / 2)$, eventually implying Eq. (27). While the first, temperature independent term in Eq. (27) describes the asymmetry of the zero LL with respect to zero energy, the second term encodes its temperature-dependent response. This term ensures that at $T=0$ the zero LL only contributes outside of the Dirac mass gap, exactly compensating its contribution to the spectral asymmetry. As expected, this property becomes softened by finite temperature effects. 